\title{
Isolation and Characterization of New Polymorphic Simple Sequence Repeat Loci in Chrysopogon aciculatus (Retz.) Trin.
}

\author{
Xinyi Zhang, Li Liao, Yang Liu, and Zhiyong Wang ${ }^{1}$ \\ Key Laboratory of Protection and Developmental Utilization of Tropical \\ Crop Germplasm Resources, Ministry of Education/College of Agriculture, \\ Hainan University, Haikou 570228, China
}

\section{Jianxiu Liu \\ Institute of Botany, Jiangsu Province and Chinese Academy of Sciences, Jiangsu Nanjing 210014, China}

Additional index words. microsatellite markers, genetic diversity, Roche 454 sequencing, Chrysopogon aciculatus (Retz.) Trin., UPGMA cluster analysis

\begin{abstract}
Chrysopogon aciculatus (Retz.) Trin. is a perennial turfgrass for its low management and resistance. To develop simple sequence repeat (SSR) markers for C. aciculatus, we used four Roche 454 pyrosequencing, combined with the magnetic bead enrichment method FIASCO (fast isolation by amplified fragment length polymorphism of sequences containing repeats) to isolate from the $C$. aciculatus. A total of 66,198 raw sequencing reads were obtained with 4289 sequences $(6.48 \%)$ were fit for primer pair design. One hundred microsatellite loci were selected to test the primer amplification efficiency in 20 accessions, and out of these, 11 loci were polymorphic. The amount of observed alleles ranged from three to six, with an average of 3.64. Nei's genetic diversity values ranged from 0.085 to 0.493 , with an average of 0.293 . Shannon's information index values ranged from 0.141 to 0.686 , with an average of 0.428 . Twenty accessions were clustered into three groups by unweighted pair-group method with arithmetic means (UPGMA). These SSR markers will provide an ideal marker system to assist with gene targeting, cultivar variety or species identification, and marker-assisted selection in C. aciculatus species.
\end{abstract}

Chrysopogon aciculatus (Retz.) Trin. is a perennial turfgrass with many features of drought resistance, shade resistance, leanness resistance, and low management. It is usually found in sunny, dry, exposed areas such as roadsides, lawns, pasture, bank of rivers, and water courses (Sun, 1995). All C. aciculatus individuals are polyploidy, with $2 n=20$ (Liao et al., 2011). In China, the wild germplasm resources of $C$. aciculatus are mainly distributed in the tropical and subtropical climate regions, such as Hainan, Guangdong, Guangxi, Yunnan, and Guizhou (Liu et al., 1998; Zheng et al., 2005). It is an excellent kind of grass for water and soil conservation often used for highway slope conservation, and also for common green land and shade turf in tropical and subtropical region of China. Previous studies of C. aciculatus have mainly focused on its morphological characteristics (Liao et al., 2011; Wagner et al.,

Received for publication 10 Nov. 2015. Accepted for publication $13 \mathrm{Jan} .2016$.

This work was supported by the National Natural Science Foundation of China (31260489).

We thank for all the reviewers' useful comments and suggestions on the structure of our manuscript. ${ }^{1}$ Corresponding author. E-mail: wangzhiyong7989@, 163.com.
1999). Liu et al. (1997) had investigated its distribution in the range of China. Bai et al. (2002) evaluated about the ecological adaptation. Reddi et al. (2010) studied pollination and seed of C. aciculatus. Ambasta and Rana (2013) studied the taxonomy of C. aciculatus. Up to date, the genetic diversity of its wild germplasms is little known. Therefore, developing a set of reliable and informative DNA markers to aid in assessing the genetic diversity of its wild germplasm resources would provide theoretical basis for the future study of breeding excellent cultivars. Microsatellite or SSR is a novel molecular genetic markers. Microsatellite has the characteristics of being a total of codominant modes, following the Mendel's law, large number, wide and uniform distribution, the high polymorphism information content, and easy to be amplified by polymerase chain reaction (PCR) (Nichols et al., 2003). It has been widely used in population genetic analysis, paternity testing, genome mapping, and marker assisted selection. FIASCO first proposed in 2002 (Zane et al., 2002) has been maturely used in many field (Chen et al., 2007; Hou et al., 2011; Wang et al., 2014; Xu et al., 2006; Yang et al., 2009; Zhang et al., 2008). However, this method is complicated to operate, and cannot obtain a large number of SSRs in one experiment. Nowadays next-generation sequencing has been the most popular approach in the research of transcription group (Li et al., 2013), such as the launch of Roche 454 pyrosequencing, has got good application for the structural variation in the genome (Alagna et al., 2009; Barakat et al., 2009; Dassanayake et al., 2009; Guo et al., 2010; Kumar et al., 2014; Swarbreck et al., 2011; Wang and Wang, 2015). We used Roche 454 pyrosequencing, combined with the magnetic bead enrichment method FIASCO, to isolate SSR markers from the C. aciculatus genome. The objectives of this study were 1) using Roche 454 pyrosequencing to isolate SSR markers from the $C$. aciculatus genome and 2) to study the genetic relationships among 20 accessions of $C$. aciculatus from China and describe the genetic variation.

\section{Materials and Methods}

Genomic DNA extraction and SSR mining. Genomic DNA was extracted using a plant genomic DNA kit (Tiangen Biotech, Beijing, China) from silica gel-dried leaves. A shotgun library was constructed following Roche protocol with about $1 \mu \mathrm{g}$ of genomic DNA. The shotgun library was further enriched by seven 5'-biotinylated probes: (AC)12, (AG)12, (AAT)8, (AGG)8, (AGC)8, (AGAT)6, (ACAG)6, according to the protocol of Toth and Gaspari (2000). Roche 454 GS-FLX+ System (Shanghai, China) was used to sequence the enriched products on a picotiter plate to search for microsatellite sequences by MISA (MIcroSAtellite identification tool) (http://pgrc.ipk-gatersleben.de/misa/). Search parameters were set as 10 repeat units for mononucleotides, six repeat units for dinucleotides, five repeat units for trinucleotides, tetranucleotides, pentanucleotides, and hexanucleotides. The maximum difference between two SSRs was set at 100 nucleotides. Primer design was conducted with Primer 3 (Rozen and Skaletsky, 2000). The PCR product size range was set at 100-400 bp and the remaining parameters were set at default values.

$P C R$ amplification and genotyping. A total of 20 individuals of $C$. aciculatus from five provinces in China (Table 1) were used to analyze the polymorphisms of the microsatellite primers. PCRs were performed in a total volume of $20 \mu \mathrm{L}$, which contained $10 \mathrm{ng}$ of genomic DNA, $0.18 \mathrm{~mm} / \mathrm{L}$ of each dNTP, $0.2 \mu \mathrm{M} / \mathrm{L}$ of each primer, $2.0 \mu \mathrm{L}$ of $10 \times$ polymerization buffer, and 1.0 unit of Taq polymerase (Tiangen Biotech, Beijing, China), using a PTC-200 Thermal Cycler (MJ Research, Watertown, MA). The PCR was conducted as follows: an initial denaturation for 5 minutes at $95{ }^{\circ} \mathrm{C}$, followed by 40 cycles of denaturation for 30 seconds at $94{ }^{\circ} \mathrm{C}$, annealing for 30 seconds at $48-60{ }^{\circ} \mathrm{C}$ (optimized for each locus, Table 2), extension for 45 seconds at $72^{\circ} \mathrm{C}$, and a final extension step of 10 minutes at $72{ }^{\circ} \mathrm{C}$.

The PCR products were electrophoresed on an $8 \%$ polyacrylamide gel and visualized by silver staining. A 100-bp DNA ladder (Takara, Dalian, China) was used as reference for estimating allele sizes.

Data analysis. Polymorphism statistics, including allele number, Nei's measure of 
gene diversity (Nei, 1973), were recorded using POPGENE v.1.3.2, software for genetic similarity analysis (Yeh and Boyle, 1996). Genetic relationships between 20 individuals of $C$. aciculatus, based on Nei's genetic distance (Nei,
1978), were examined using the UPGMA in NTSYS-pc 2.10e (Rohlf, 2000). Shannon's information index (I) were used to construct a UPGMA dendrogram within the sequential agglomerative hierarchical nested cluster analysis

Table 1. Details of locations of 86 Chrysopogon aciculatus accessions.

\begin{tabular}{|c|c|c|c|c|c|}
\hline No. & Accession no. & Origin & No. & Accession no. & Origin \\
\hline 1 & CA01 & Haikou, Hainan & 44 & CA46 & Qiongzhong, Hainan \\
\hline 2 & $\mathrm{CA} 02$ & Qionghai, Hainan & 45 & CA47 & Longzhou, Guangxi \\
\hline 3 & CA03 & Dongfang, Hainan & 46 & CA48 & Changtai, Fujian \\
\hline 4 & CA04 & Qionghai, Hainan & 47 & CA49 & Pinghe, Fujian \\
\hline 5 & CA05 & Qionghai, Hainan & 48 & CA50 & Wengyuan, Guangxi \\
\hline 6 & CA06 & Qionghai, Hainan & 49 & CA51 & Lingao, Hainan \\
\hline 7 & CA07 & Danzhou, Hainan & 50 & CA52 & Guiping, Guangxi \\
\hline 8 & CA08 & Qionghai, Hainan & 51 & CA53 & Qinzhou, Guangxi \\
\hline 9 & CA09 & Wanning, Hainan & 52 & CA54 & Hepu, Guangxi \\
\hline 10 & CA10 & Wanning, Hainan & 53 & CA55 & Qiongzhong, Hainan \\
\hline 11 & CA11 & Wanning, Hainan & 54 & CA56 & Hepu, Guangxi \\
\hline 12 & CA12 & Danzhou, Hainan & 55 & CA57 & Hepu, Guangxi \\
\hline 13 & CA14 & Dingan, Hainan & 56 & CA58 & Wuzhishan, Hainan \\
\hline 14 & CA15 & Danzhou, Hainan & 57 & CA59 & Qiongzhong, Hainan \\
\hline 15 & CA16 & Dingan, Hainan & 58 & CA60 & Wuzhishan, Hainan \\
\hline 16 & CA18-1 & Danzhou, Hainan & 59 & CA61 & Wuzhishan, Hainan \\
\hline 17 & CA18-2 & Danzhou, Hainan & 60 & CA62 & Wuzhishan, Hainan \\
\hline 18 & CA19 & Qionghai, Hainan & 61 & CA63 & Wuzhou, Guangxi \\
\hline 19 & CA20 & Haikou, Hainan & 62 & CA64 & Qiongzhong, Hainan \\
\hline 20 & CA21 & Dongfang, Hainan & 63 & CA65 & Wuzhou, Guangxi \\
\hline 21 & CA22 & Changjiang, Hainan & 64 & CA66 & Qiongzhong, Hainan \\
\hline 22 & CA23 & Sanya, Hainan & 65 & CA67 & Shanghang, Fujian \\
\hline 23 & CA25 & Cenxi, Guangxi & 66 & CA68 & Wuzhou, Guangxi \\
\hline 24 & CA26 & Yingde, Guangdong & 67 & CA69 & Hepu, Guangxi \\
\hline 25 & CA27 & Guigang, Guangxi & 68 & CA70 & Xuwen, Guangdong \\
\hline 26 & CA28 & Beihai, Guangxi & 69 & CA71 & Maoming, Guangdong \\
\hline 27 & CA29 & Sanya, Hainan & 70 & CA72 & Bobai, Guangxi \\
\hline 28 & CA31 & Chengmai, Hainan & 71 & CA73 & Suixian, Guangdong \\
\hline 29 & CA31 & Chengmai, Hainan & 72 & CA74 & Hepu, Guangxi \\
\hline 30 & CA32 & Laibin, Guangxi & 73 & CA75 & Puer, Yunnan \\
\hline 31 & CA33 & Zhaoqing, Guangdong & 74 & CA76 & Zhenyuan, Yunnan \\
\hline 32 & CA34 & Tunchang, Hainan & 75 & CA77 & Ninger, Yunnan \\
\hline 43 & CA45 & Daxin, Guangxi & 86 & CA89 & Baisha, Hainan \\
\hline
\end{tabular}

module of NTSYS (taxonomy and multivariate analysis system) (Sneath and Sokal, 1973). Finally, a Mantel test between the geographic and genetic distances have been carried out using $\mathrm{COPH}$ (cophenetic values) and MXCOP (matrix comparison plot) modules in NTSYS-pc software (Mantel, 1967).

\section{Results and Discussion}

A total of 66,198 raw sequencing reads were obtained. 17,161 sequences $(25.92 \%)$ contained SSRs and only 4289 sequences $(6.48 \%)$ were fit for primer pair design. The properties of mono-, di-, tri-, tetra-, penta-, and hexaploid were $6.35 \%, 47 \%, 41.42 \%, 4.85 \%, 0.05 \%$, and $0.35 \%$, respectively. We randomly selected 100 microsatellite sequences to design primer pairs and tested. These sequences were deposited in GenBank (KT946824-KT946834). Twenty-six primer pairs, which could successful amplify target fragments, were tested for polymorphisms in 20 accessions collected from five provinces in China (Table 1); 11 loci were polymorphic and 15 displayed monomorphic. The genetic diversity parameters of each material were represented in Table 2. The amount of observed alleles ranged from 3 (IJ3Q0KF02FQL5F, IJ3Q0KF02GCT8C, IJ3Q0KF02GKSXP， IJ3Q0KF02IC1FO, IJ3Q0KF02IWEAL, IJ3Q0KF02FH3GM) to 6 (IJ3Q0KF02IILLR), with an average of 3.64. Nei's genetic diversity values ranged from 0.085 (IJ3Q0KF02FQL5F) to 0.493 (IJ3Q0KF02H9WIF), with an average of 0.293 . Shannon's information index values ranged from 0.141 (IJ3Q0KF02FQL5F) to 0.686 (IJ3Q0KF02H9WIF), with an average of 0.428 . This result suggested that 20

Table 2. Primer sequences and characterization of 11 microsatellite loci isolated from Chrysopogon aciculatus.

\begin{tabular}{|c|c|c|c|c|c|c|c|c|}
\hline Primer & Primer sequence $\left(5^{\prime}-3^{\prime}\right)$ & Repeat motif & $\mathrm{Ta}^{\mathrm{z}}\left({ }^{\circ} \mathrm{C}\right)$ & $\begin{array}{l}\text { Allele size } \\
\quad \text { (bp) }\end{array}$ & $\mathrm{A}^{y}$ & $\mathrm{H}_{\mathrm{E}}{ }^{\mathrm{x}}$ & $\mathrm{I}^{\mathrm{w}}$ & $\begin{array}{c}\text { GenBank } \\
\text { accession no. }\end{array}$ \\
\hline$\overline{\mathrm{IJ} 3 \mathrm{Q} 0 \mathrm{KF} 02 \mathrm{HEEVY}}$ & $\begin{array}{l}\text { F:AGGAGGAAGAGGAGGACGAG } \\
\text { R:CCATGAGGAGGAAGAGCAAG }\end{array}$ & (GGA)12 & 59.9 & $166-364$ & 4 & 0.383 & 0.567 & KT946824 \\
\hline IJ3Q0KF02JB94X & $\begin{array}{l}\text { F:ATCCCAGCATATCCAAATCG } \\
\text { R:CACCACCAATCCACCATGTA }\end{array}$ & (AAG)8acgacgacgacc(AAG)6 & 60.1 & $86-315$ & 4 & 0.158 & 0.246 & KT946825 \\
\hline IJ3Q0KF02IILLR & $\begin{array}{l}\text { F:CGCAAGAACTCTGGAGGAAC } \\
\text { R:TCGTAATAGCTGCACCATGC }\end{array}$ & (AG)24 & 59.9 & $117-318$ & 6 & 0.306 & 0.459 & KT946826 \\
\hline IJ3Q0KF02H9WIF & $\begin{array}{l}\text { F:CCCCTCCATCACCATAGCTT } \\
\text { R:CTGCAAGCCAACAGAAACAA }\end{array}$ & (TTG) 12 & 61.2 & $178-395$ & 4 & 0.493 & 0.686 & KT946827 \\
\hline IJ3Q0KF02FQL5F & $\begin{array}{l}\text { F:CATCGCAGATTGTCCTCTCA } \\
\text { R:GGAGCATTGAAGAGTGAGGC }\end{array}$ & $\begin{array}{l}\text { (AAG)8gacaagagagagaagaagaaca } \\
\text { agaaggagaagagaagaaggttgagtac } \\
\text { (AAG) } 5\end{array}$ & 59.9 & $164-433$ & 3 & 0.085 & 0.141 & KT946828 \\
\hline IJ3Q0KF02FOQLC & $\begin{array}{l}\text { F:GCCAATGACGTTGAACACC } \\
\text { R:TCAGCAGATGAGCAGAGTCG }\end{array}$ & (TCT) 19 & 59.9 & $111-295$ & 4 & 0.204 & 0.297 & KT946829 \\
\hline IJ3Q0KF02GCT8C & $\begin{array}{l}\text { F:CGGATTCATGTGACCTGTGT } \\
\text { R:TTTGGTGATTTCTTCGTCCC }\end{array}$ & $(\mathrm{CA}) 16(\mathrm{TA}) 6$ & 59.4 & $213-409$ & 3 & 0.460 & 0.652 & KT946830 \\
\hline IJ3Q0KF02GKSXP & $\begin{array}{l}\text { F:GCCGACAGGAATAGGACAGA } \\
\text { R:ACACCATCCTGGAACCCATA }\end{array}$ & (AC)27 & 60.2 & 104-372 & 3 & 0.165 & 0.229 & KT946831 \\
\hline IJ3Q0KF02IC1FO & $\begin{array}{l}\text { F:CACGTGATCTTGGACAATGG } \\
\text { R:TCACACTATAAAAGAGCGAGCG }\end{array}$ & (TGAGA)5(GAT) $9^{*}$ & 59.7 & $98-348$ & 3 & 0.297 & 0.465 & KT946832 \\
\hline IJ3Q0KF02IWEAL & $\begin{array}{l}\text { F:CCTGATCGATCTCGACACAA } \\
\text { R:TTTTCTGTGTCTCTGTGTGTGTG }\end{array}$ & $\begin{array}{l}\text { (AG)6ggagaaagaaaacacacacattc } \\
\text { (AG)6 }\end{array}$ & 59.8 & $41-300$ & 3 & 0.472 & 0.665 & KT946833 \\
\hline IJ3Q0KF02IRPRC & $\begin{array}{l}\text { F:TCACTTCATCGCGAATTGTC } \\
\text { R:TGTAGCATGCCAATGAGGTC }\end{array}$ & $\begin{array}{l}\text { (AGA)5aggagaagaaagagaaaaagtt } \\
\text { tgagtac(AAG)5 }\end{array}$ & 59.7 & $158-541$ & 3 & 0.198 & 0.297 & KT946834 \\
\hline
\end{tabular}

${ }^{2}$ Polymerase chain reaction annealing temperature.

'Number of alleles.

${ }^{\mathrm{x}}$ Nei's measure of gene diversity.

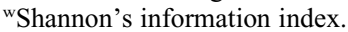




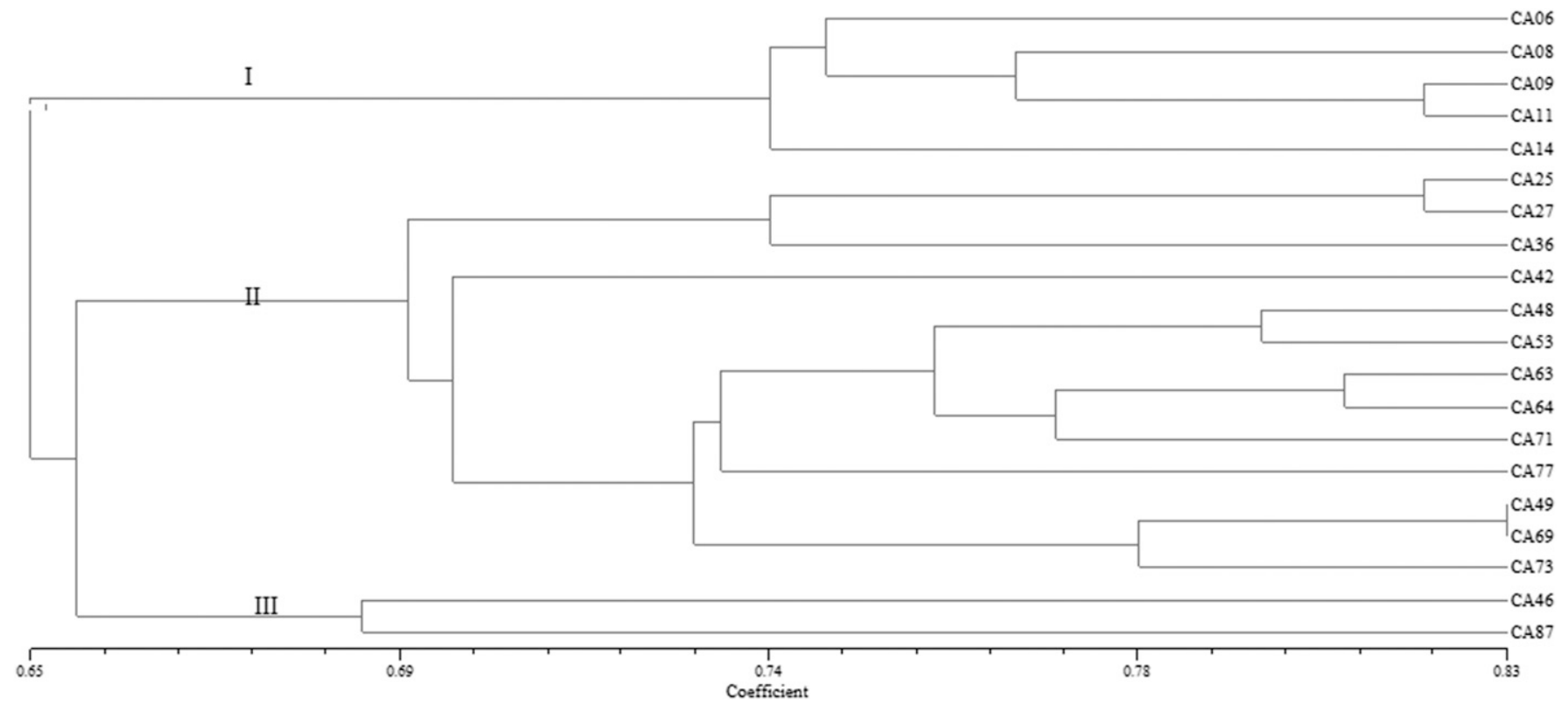

Fig. 1. Unweighted pair-group method with arithmetic means tree illustrating the genetic relationships between 20 accessions of Chrysopogon aciculatus based on Nei's genetic distance.

accessions possessed a great amount of variation and represented the accessions of $C$. aciculatus possessed great genetic diversity.

We could know from the UPGMA tree, individuals CA06, CA08, CA09, CA11, and CA14 had closed phylogenetic relationships in group I. All of these materials come from Hainan Province. Group II included all individuals coming from Guangxi Province (CA25, CA27, CA36, CA53, CA63, CA64, and CA69), Guangdong Province (CA71 and CA73), Fujian Province (CA49 and CA48), Yunnan Province (CA77), and one individual from Hainan Province (CA42). CA46 and CA87 in group III (Fig. 1) were both coming from Qiongzhong, Hainan. CA49 (Pinghe, Fujian) and CA69 (Hepu, Guangxi) had the closest phylogenetic relationship. UPGMA showed the materials from the same area were more easily clustered together. This phenomenon also mentioned in other studies (Budak et al., 2004; Tani et al., 1998; Wang and Wang, 2015; Wang et al., 2010, 2013). C. aciculatus has been characterized as a species with strong vegetative reproduction, and through human activities it is easily to be taken to different place, and it would have a high level of genetic diversity with extension, and reproduction. Introgression may lead to cross clustering among materials from different regions. In this study, eight accessions, which came from Hainan (CA06, CA08, CA09, CA11, CA14, CA42, CA46, and CA87), were clustered into different groups. Actually, they exhibited great difference on turf characteristics. CA46 and CA 87 were clustered in group III, both two accessions have higher length of leaves both stolons and upright stems, length of internode, and relatively larger dense inflorescence. The five accessions in group I (CA06, CA08, CA09, CA11, and CA14) demonstrated opposing characteristics to those of group III. CA42 from Haikou, Hainan were clustered in group II which included 13 accessions from 5 provinces. Characteristics of CA42 and other materials in group II were generally in-between those of group I and group III. The relationship between their genetic distance and geographical origins were complex. We should make full use of excellent $C$. aciculatus germplasm gathered in different types or subtypes during the selection of crossbreeding parents, without being limited to geographical factors.

We also used the COPH and MXCOP module calculate goodness-of-fit generated between the geographic and genetic distances, the result of Mantel test was $r=$ 0.7372 . This turned out that the geographic and genetic distances correlated positively.

In conclusion, this study was the first to isolate and use microsatellite markers in C. aciculatus. The studies indicated that there was great genetic diversity among C. aciculatus germplasms. This first set of microsatellite markers developed for $C$. aciculatus was promised to be useful in the studies of cultivars identification, genetic relationship, and assessment of wild germplasm resources.

\section{Literature Cited}

Alagna, F., N. D'Agostino, L. Torchia, M. Servili, R. Rao, M. Pietrella, G. Giuliano, M.L. Chiusano, L. Baldoni, and G. Perrotta. 2009. Comparative 454 pyrosequencing of transcripts from two olive genotypes during fruit development. BMC Genomics 10:399.

Ambasta, N. and N.K. Rana. 2013. Taxonomical study of Chrysopogon aciculatus (Retz.) Trin. a significant grass of Chauparan, Hazaribag (Jharkhand). Sci. Res. Rptr. 3:27.

Bai, C.J., G.D. Liu, and J.S. Wei. 2002. The selection and utilization of Zoysia matrella cv. Huanan. Acta Agrestia Sinica 10:112-117.

Barakat, A., D.S. DiLoreto, Y. Zhang, C. Smith, K. Baier, W.A. Powell, N. Wheeler, R. Sederoff, and J.E. Carlson. 2009. Comparison of the transcriptomes of American chestnut (Castanea dentata) and Chinese chestnut (Castanea mollissima) in response to the chestnut blight infection. BMC Plant Biol. 9:51.

Budak, H., R.C. Shearm, I. Parmaksiz, and I. Dweikat. 2004. Comparative analysis of seeded and vegetative biotype buffalograsses based on phylogenetic relationship using ISSRs, SSRs, RAPDs, and SRAPs. Theor. Appl. Genet. 109: 280-288.

Chen, G.Q., L.F. Li, G. Hao, S.H. Shi, and X.J. Ge. 2007. Characterization of seven genomic and one dbEST-derived microsatellite loci in the river mangrove Aegiceras corniculatum (Myrsinaceae). Conserv. Genet. 9:449-451.

Dassanayake, M., J.S. Haas, H.J. Bohnert, and J.M. Cheeseman. 2009. Shedding light on an extremophile lifestyle through transcriptomics. New Phytol. 183:764-775.

Guo, S., Y. Zheng, J.G. Joung, S. Liu, Z. Zhang, O.R. Crasta, W.S. Bruno, X. Yong, H. Sanwen, and J.F. Zhang. 2010. Transcriptome sequencing and comparative analysis of cucumber flowers with different sex types. BMC Genomics 11:384.

Hou, X.G., D.L. Guo, S.P. Cheng, and J.Y. Zhang. 2011. Development of thirty new polymorphic microsatellite primers for Paeonia suffruticosa. Biol. Plant. 55:708-710.

Kumar, S., N. Shah, V. Garg, and S. Bhatia. 2014. Large scale in-silico identification and characterization of simple sequence repeats (SSRs) from denovo assembled transcriptome of Catharanthus roseus (L.). G. Don. Plant Cell Rpt. 33:905-918.

Li, X.B., L. Xiang, J. Luo, B.L. Hu, S.P. Tian, M. Xie, and C. Sun. 2013. The strategy of RNAseq, application and development of molecular marker derived from RNA-seq. Chinese J. Cell Biol. 35:1-7.

Liao, L., C.J. Bai, X.L. Guo, and Z.Y. Wang. 2011. Morphological diversity of Chrysopogon aciculatus. Chinese J. Trop. Crops 32:2042-2047.

Liu, J.X., S.A. He, and Y.D. Liu. 1997. Characteristics and economic value of warm season turfgrasses in east China. Grassland China 4:62-66.

Liu, J.X., Y.D. Liu, S.A. He, and S.L. Chen. 1998. Species diversity of Chinese warm season 
turfgrass and the characteristic of geography distribution. Acta Agrestia Sinca 6:45-52.

Mantel, N. 1967. The detection of disease clustering and a generalized regression approach. Cancer Res. 27:209-220.

Nei, M. 1973. Analysis of gene diversity in subdivided populations. Proc. Natl. Acad. Sci. USA 70:3321-3323.

Nei, M. 1978. Estimation of average heterozygosity and genetic distance from a small number of individuals. Genetics 76:379-390.

Nichols, K.M., W.P. Young, B.D. Robison, C. Rexroad, M. Noakes, R.B. Phillips, P. Bentzen, I. Spies, K. Knudsen, F.W. Allendorf, B.M. Cunningham, J. Brunelli, H. Zhang, S. Ristow, R. Drew, K.H. Brown, P.A. Wheeler, and G.H. Thorgaard. 2003. A consolidated linkage map for rainbow trout (Oncorhynchus mykiss). Anim. Genet. 34:102-115.

Reddi, C.S., N.S.N. Raju, and M.V.S. Rao. 2010. Pollination and seed set in tropical wetland grasses. Nord. J. Bot. 28:354-365.

Rohlf, F.J. 2000. NTSYS-pc. Numerical taxonomy and multivariate analysis system. Version 2.1. Exeter Publ., Setauket, NY.

Rozen, S. and H. Skaletsky. 2000. Primer3 on the WWW for general users and for biologist programmers, p. 365-386. In: S. Krawetz and S. Misener (eds). Bioinformatics methods and protocols: Methods in molecular biology. Humana Press, Totowa, NJ.

Sneath, P.H.A. and R.R. Sokal. 1973. Numerical taxonomy. Freeman, San Francisco, CA.
Sun, J.X. 1995. Turf science. China Agriculture Press, Beijing, China.

Swarbreck, S.M., E.A. Lindquist, D.D. Ackerly, and G.L. Andersen. 2011. Analysis of leaf and root transcriptomes of soil-grown Avena barbata plants. Plant Cell Physiol. 52:317332.

Tani, N., N. Tomaru, Y. Tsumura, A. Masayuki, and O. Kihachiro. 1998. Genetic structure within a Japanese stone pine (Plnus pumila Regal) population on Mt. Aino-dake in Central Honshu. Plant Res. 111:7-15.

Toth, G. and Z.J. Gaspari. 2000. Microsatellites in different eukaryotic genomes: survey and analysis. Genome Research 10:967-981.

Wang, Z., K.E. Kenworthy, and Y.Q. Wu. 2010. Genetic diversity of common carpetgrass revealed by amplified fragment length polymorphism markers. Crop Sci. 50:1366-1374.

Wang, Z.Y., L. Liao, X.J. Yuan, H.L. Guo, A.G. Guo, and J.X. Liu. 2013. Genetic diversity analysis of Cynodon dactylon (bermudagrass) accessions and cultivars from different countries based on ISSR and SSR markers. Biochem. Syst. Ecol. 46:108-115.

Wang, X.L. and Z.Y. Wang. 2015. Genetic diversity of carpetgrass germplasm based on simple sequence repeat markers. HortScience 50:797-800.

Wang, M., F. Xue, P. Yang, X.Y. Duan, Y.L. Zhou, C.Y. Shen, G.Z. Zhang, and B.T. Wang. 2014. Development of SSR markers for a phytopathogenic fungus, Blumeria graminis f.sp. tritici, using a FIASCO protocol. Integr. Agr. 13:100-104.

Wagner, W.L., D.R. Herbst, and S.H. Sohmer. 1999. Manual of the flowering plants of Hawaii, p. 1516-1517. Revised edition. University of Hawaii Press, Honolulu, HI.

Xu, Y.Q., Y. Wang, Z.Z. Li, Z.X. Bao, J.F. Zhou, and H.W. Huang. 2006. Characterization of polymorphic microsatellite loci in a traditional Chinese medicinal plant, Gastrodia elata. Mol. Ecol. Notes 6:316-318.

Yang, J.B., J. Yang, H.T. Li, Y. Zhao, and S.X. Yang. 2009. Isolation and characterization of 15 microsatellite markers from wild tea plant (Camellia taliensis) using FIASCO method. Conserv. Genet. 10:1621-1623.

Yeh, F.C. and T.J.B. Boyle. 1996. Population genetic analysis of co-dominant and dominant markers and quantitative traits. Belg. J. Bot. 129:157.

Zane, L., L. Bargelloni, and T. Patarnello. 2002. Strategies for microsatellite isolation: A review. Mol. Ecol. 11:1-16.

Zhang, J., X. Yao, X. Wei, L. Chen, and M. Jiang. 2008. Development and characterization of 14 polymorphic microsatellite loci in the endangered tree Euptelea pleiospermum (Eupteleaceae). Mol. Ecol. Resour. 8:314-316.

Zheng, Y.Z., J.B. Xi, and Z.Y. Yang. 2005. Studies on distribution and morphological variation of wild grass Chrysopogon aciculatus collected from the tropics and subtropics of China. Acta Agrestia Sin. 3:117-122. 